\title{
PRIMENESS AND SUMS OF TANGLES
}

\author{
MARIO EUDAVE-MUÑOZ
}

\begin{abstract}
We consider knots and links obtained by summing a rational tangle and a prime tangle. For a given prime tangle, we show that there are at most three rational tangles that will induce a composite or splittable link. In fact, we show that there is at most one rational tangle that will give a splittable link. These results extend Scharlemann's work.
\end{abstract}

1. Introduction. A tangle $(B, t)$ is a pair that consists of a 3 -ball $B$ and a pair of disjoint arcs $t$ properly embedded in $B$. Two tangles are equivalent if there is a homeomorphism $h$ between the pairs. Two tangles are equal if there is a homeomorphism $h:(B, t) \rightarrow\left(B, t^{\prime}\right)$ of pairs such that $h \mid \partial B=\mathrm{id}$.

A trivial tangle is a tangle equivalent to the standard pair $\left(D^{2} \times I,\{u, v\} \times I\right)$, $u, v$ in the interior of $D^{2}, u \neq v$. A rational tangle is an element of an equivalence class of trivial tangles under the equality relation; there is a one to one correspondence between the rational tangles and $\mathbf{Q} \cup\{1 / 0\}$ (see $\left[\mathbf{C}, \mathbf{M}_{1}\right]$ ). Let $(B, p / q)$ denote the tangle determined by $p / q$, the standard pair is $(B, 1 / 0)$; we denote the homeomorphism between $(B, 1 / 0)$ and $(B, p / q)$ as trivial tangles by $h_{p / q}:(B, 1 / 0) \rightarrow(B, p / q)$.

Let $J$ be a meridian of $(B, 1 / 0)$ as in Figure 1 . Let $(B, p / q)$ and $(B, r / s)$ be two rational tangles. The distance between them denoted $d((B, p / q),(B, r / s))$ or more simply $d(p / q, r / s)$, is defined to be the minimum (over all the representatives) of $\frac{1}{2} \#\left(h_{p / q}(J) \cap h_{r / s}(J)\right)$. It can be shown that $d(p / q, r / s)=|p s-q r|$.

A tangle $(B, t)$ is prime if has the following properties: (a) It has no local knots, that is, any $S^{2}$ which meets $t$ transversely in two points, bounds in $B$ a ball meeting $t$ in an unknotted spanning arc; (b) there is no disc properly embedded in $B$ which separates the strings of $(B, t)$. We refer to $[\mathbf{L}]$ for definitions and facts about tangles not found here.

Let $k$ be a knot or link in $S^{3} . \quad k$ is splittable if there is a $S^{2}$ in $S^{3}-k$ that separates the components of $k . k$ is composite if there is a $S^{2}$ in $S^{3}$, which meets $k$ transversely in two points, such that neither of the closures of the components of $S^{3}-S^{2}$ meets $k$ in a single unknotted spanning arc. $k$ is prime if it is neither splittable, nor composite, nor trivial.

To sum a rational tangle $\left(B^{\prime}, r\right)$ to a tangle $(B, t)$ means the following: take an embedding of $(B, t)$ into $S^{3}$ and also an embedding of $\left(B^{\prime}, 1 / 0\right)$ and join them as in Figure 2(a), now replace $\left(B^{\prime}, 1 / 0\right)$ by $h_{r}\left(\left(B^{\prime}, 1 / 0\right)\right)=\left(B^{\prime}, r\right)$ as in Figure 2(b).

Received by the editors February 26, 1987. Presented at the XIX congress of the Mexican Mathematical Society, held at Guadalajara, Mexico, on November 16-21, 1986.

1980 Mathematics Subject Classification (1985 Revision). Primary 57M25; Secondary 57N10.

Key words and phrases. Prime tangle, rational tangle, prime knot and link, composite knot and link, splittable link. 


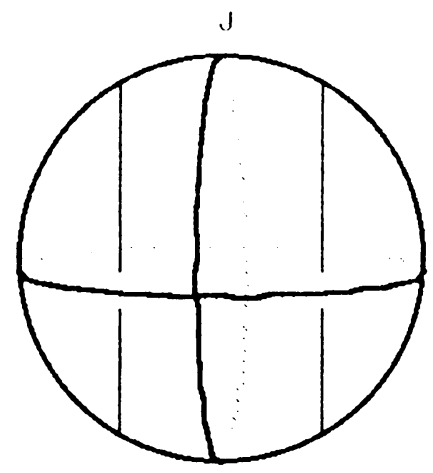

(B, 1/0)

FIGURE 1

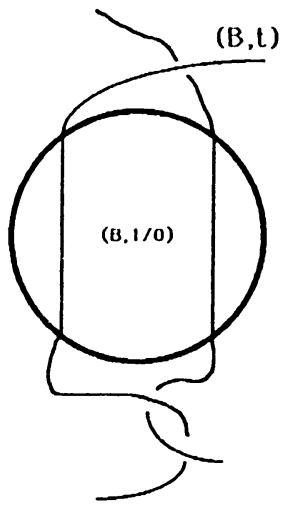

(a)

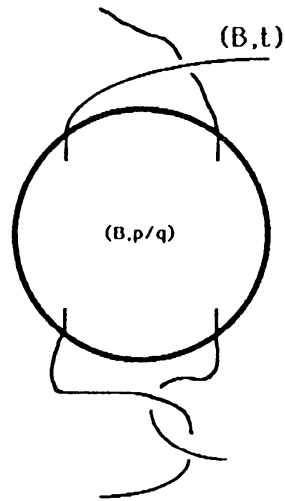

(b)

FIGURE 2

Let $(B, t)$ be any tangle, fix an embedding of $(B, t)$ in $S^{3}$. Let $\left(B^{\prime}, r_{i}\right), i=1,2$, be two rational tangles, and let $k_{i}$ be the knot or link obtained by $\operatorname{summing}(B, t)$ and $\left(B^{\prime}, r_{i}\right)$. Our results are the following:

THEOREM 1. Let $(B, t)$ be a prime tangle. If $k_{1}$ and $k_{2}$ are composite then $d\left(r_{1}, r_{2}\right) \leq 1$.

THEOREM 2. Let $(B, t)$ be a prime tangle. If $k_{1}$ is composite and $k_{2}$ is splittable, then $d\left(r_{1}, r_{2}\right) \leq 1$.

THEOREM 3. Let $(B, t)$ be any tangle. If $k_{1}$ and $k_{2}$ are splittable, then $r_{1}=r_{2}$.

In this same direction there are the following results.

THEOREM 4. Let $(B, t)$ be a prime tangle. If $k_{1}$ and $k_{2}$ are trivial knots, then $r_{1}=r_{2}\left[\mathbf{B S}_{1}, \mathbf{B S}_{2}\right]$.

THEOREM 5. Let $(B, t)$ be any tangle. If $k_{1}$ is a trivial knot and $k_{2}$ is splittable, then $d\left(r_{1}, r_{2}\right) \leq 1$, and $(B, t)$ is a trivial tangle $\left[\mathbf{S}_{1}\right]$.

THEOREM 6. Let $(B, t)$ be a prime tangle. If $k_{1}$ is a trivial knot and $k_{2}$ is composite, then $d\left(r_{1}, r_{2}\right) \leq 1[\mathbf{E}]$. 
COROLLARY 1. Given a prime tangle $(B, t)$ there are at most three rational tangles $\left(B^{\prime}, r_{i}\right), i=1,2,3$, such that the knot or link $k_{i}$ that results summing $(B, t)$ and $\left(B^{\prime}, r_{i}\right)$ is nonprime. Furthermore $d\left(r_{i}, r_{j}\right) \leq 1$.

Theorems 1 and 6 and some results about branched double covers of $S^{3}$ branched over a knot or link (see $\left[\mathbf{M}_{2}, \mathbf{K T}, \mathbf{B}\right]$ ) imply the following corollary.

COROLLARY 2. Let $k$ be a strongly invertible knot in $S^{3}$, and $M(k, r)$ the manifold obtained by doing surgery with coefficient $r$ on $k$. If $M\left(k, r_{1}\right)$ and $M\left(k, r_{2}\right)$ are reducible then $r_{1}$ and $r_{2}$ are integers and $\left|r_{1}-r_{2}\right| \leq 1$. If $k$ is also amphicheiral, then $M(k, r)$ is irreducible for all coefficients $r$.

W. B. R. Lickorish conjectured in $[\mathbf{L}]$ that given a prime tangle there is at most one rational tangle such that summing gives a nonprime knot; but S. A. Bleiler [B] found counterexamples, and he conjectured that given a prime tangle there is at most one rational tangle in each 'string attachment class' such that summing gives a nonprime knot or link. The truth of this conjecture is a consequence of Corollary 1. It can be observed that if for a given prime tangle there are three rational tangles such that summing yields three nonprime knots or links, then two of them must be knots and the third must be a link. It is unknown if there is a prime tangle that admits three distinct rational tangles such that summing yields three nonprime knots or links.

Theorem 6 is a generalization of Scharlemann's theorem "Unknotting number one knots are prime" $\left[\mathbf{S}_{2}\right]$. In [GL] it is proved that for any knot $k$ in $S^{3}$ the manifold $M(k, r)$ can be reducible only if $r$ is an integer; this implies Theorem 6 .

Theorems 1-6 are best possible, as is shown in the prime tangles of Figure 3. Abusing the terminology of Conway $[\mathbf{C}]$, the tangle 3(a) has 'numerator' the unknot and 'denominator' $33_{1} \# 4_{1}$, the tangle $3(\mathrm{~b})$ has numerator the square knot and denominator $33_{1} \# 9_{37}$, and the tangle $3(\mathrm{c})$ has numerator the square knot and denominator the unlink.

In $\S \S 2,3,4$, we prove Theorems $1,2,3$ respectively. The techniques used here to prove the theorems are globally much the same as those of $\left[\mathbf{S}_{1}\right.$ and $\left.\mathbf{S}_{2}\right]$. The argument consists in converting the problem into a combinatorial problem on planar graphs, and contrasts conclusions based on the topology of the underlying problem with conclusions based on the combinatorics of the graph. We refer frequently to $\left[\mathbf{S}_{1}\right.$ and $\left.\mathbf{S}_{2}\right]$, and their arguments are indispensable for the understanding of this paper.

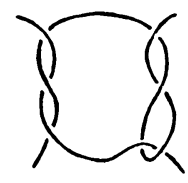

(a)

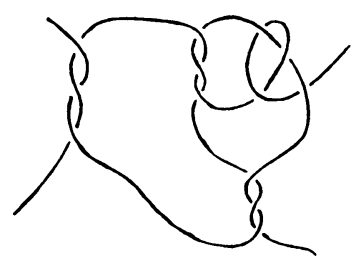

(b)

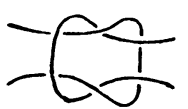

(c) 


\section{Main topological and combinatorial arguments.}

2.1 In this section we prove Theorem 1 .

Let $(B, t)$ be a prime tangle, and $\left(B^{\prime}, r_{1}\right),\left(B^{\prime}, r_{2}\right)$ two rational tangles, such that $r=d\left(r_{1}, r_{2}\right) \geq 2$. In this section we use the indices $a, b$ to denote 1 or 2 , with the convention that when used together $\{a, b\}=\{1,2\}$.

Let $k_{a}$ be the knot or link obtained by summing $(B, t)$ and $\left(B^{\prime}, r_{a}\right)$. Suppose that $k_{a}$ is composite, that is, there is a $S^{2}$ that meets $k_{a}$ in two points, such that neither of the closures of the two components of $S^{3}-S^{2}$ meets $k_{a}$ in an unknotted spanning arc. Suppose that $k_{a}$ is not a splittable link, we consider this case in $\S \S 3$ and 4. We can suppose the following, after isotopies: (a) the strings of $\left(B^{\prime}, r_{a}\right)$ are contained in $\partial B^{\prime}$, this can be done because $\left(B^{\prime}, r_{a}\right)$ is a trivial tangle; (b) $S^{2}$ meets $k_{a}$ on the strings of $(B, t)$; (c) the intersections of $S^{2}$ and $\partial B$ are all essential circles in $\partial B$-\{strings of $\left.\left(B^{\prime}, r_{a}\right)\right\}$, such that each of these circles is the boundary of a disc in $S^{2}$ whose interior does not meet $\partial B$. Let $S_{a}$ be a sphere in $S^{3}$, with the above mentioned properties such that the number of intersection circles in $S_{a} \cap \partial B$ is minimized.

Now let $P_{a}=B \cap S_{a}$, hence $P_{a}$ is a planar surface in $B$. $\partial P_{a}$ is formed of $n_{a}$ circles, parallel to $h_{r}(J)$ denoted by $a_{1}, a_{2}, \ldots, a_{n_{a}}$, labelled so that $a_{i}$ and $a_{i+1}$ cobound an essential annulus contained in $\partial B$-\{strings of $\left.\left(B^{\prime}, r_{a}\right)\right\}$ whose interior does not meet $S_{a}$, for $1 \leq i \leq n_{a}-1$. The points of $P_{a} \cap k_{a}$ are denoted $a_{+}$and $a_{-}$.

Let $a_{i}$ and $b_{j}$ be components of $\partial P_{a}$ and $\partial P_{b}$, respectively. We can suppose that $\#\left(a_{i} \cap b_{j}\right)$ is minimum; that is, equal to $2 r$. The circle $a_{i}$ meets circles of $\partial P_{b}$ as follows: first it meets $b_{1}$, then $b_{2}, \ldots, b_{n_{b}}$, then $b_{n_{b}}, \ldots, b_{1}$, then again $b_{1}, \ldots, b_{n_{b}}$, and so on successively until we return to the starting point. $b_{j}$ meets $\partial P_{a}$ similarly, see Figure 4. Label the points of intersection between $a_{i}$ and $b_{j}$ with $j$ in $a_{i}$, and with $i$ in $b_{j}$.

The intersection of $k_{a}$ and $k_{b}$ consists of two arcs, the strings of $(B, t)$, together with $2 r$ points, transversal crossings on $\partial B$. $k_{b}$ meets $P_{a}$ in the following points: $a_{+}$and $a_{-}$, and $2 r$ points in each one of the components of $\partial P_{a}$, the latter occur between the labels $1-1$ and $n_{b}-n_{b}$.

2.2 Claim. Both $P_{1}$ and $P_{2}$ are incompressible in $B$-\{strings of $\left.(B, t)\right\}$.

ProOf. Suppose that $P_{a}$ is compressible, and let $D$ be a compression disc. If $\partial D$ is essential in $S_{a}-\left\{a_{+}, a_{-}\right\}$then do disc surgery on $S_{a}$ with $D$, giving a sphere that meets $k_{a}$ in one point, but this is impossible because $S^{3}$ is irreducible. If $\partial D$ is not essential in $S_{a}-\left\{a_{+}, a_{-}\right\}$, because $k_{a}$ is not splittable, an isotopy of $S_{a}$ reduces $\#\left(S_{a} \cap \partial B\right)$, contradicting minimality. This completes the proof.

$P_{a} \cap P_{b}$ consists of arcs and circles, and by the incompressibility of $P_{a}$ and $P_{b}$, it can be supposed that all the intersection circles are essential in both $P_{a}$ and $P_{b}$.

ClaIM. There is no intersection circle between $P_{a}$ and $P_{b}$, such that one of the discs determined by it in $S_{a}$ has in its interior $a_{+}\left(a_{-}\right)$but has neither $a_{-}\left(a_{+}\right)$nor any of the $a_{i}$ 's.

PROOF. Suppose there is one such curve, take an innermost, let this be $c$, and let $D$ be the disc determined by $c$ in $S_{a}$ as above; look at $c$ in $S_{b}$, if $c$ is not essential in $S_{b}-\left\{b_{+}, b_{-}\right\}$we can construct a sphere meeting $k_{b}$ transversely in one point, but this is impossible. If $c$ is essential in $S_{b}-\left\{b_{+}, b_{-}\right\}$, there are two possibilities: 


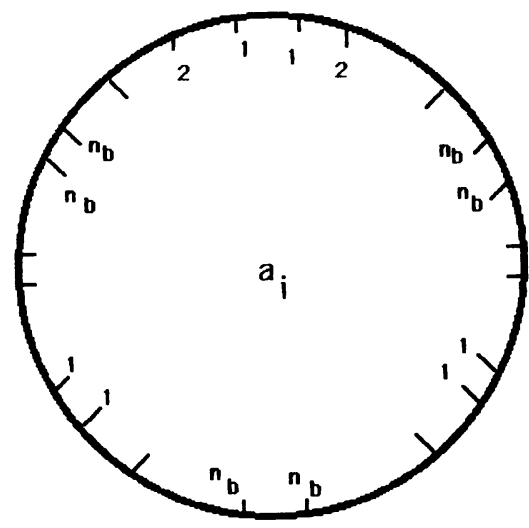

labelling about $a_{i}$

FIGURE 4

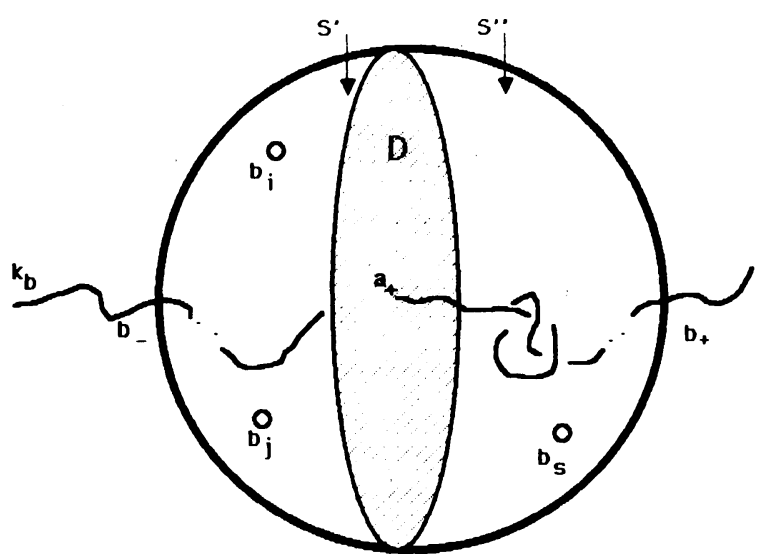

FiguRE 5

(a) $c$ is the boundary of a disc in $S_{b}$ that contains in its interior $b_{+}$(or $b_{-}$) but it does not contain a $b_{j}$.

In this case, since $(B, t)$ is a prime tangle, an isotopy removes the intersection.

(b) The two discs determined by $c$ in $S_{b}$ have in their interior some of the $b_{j}$ 's.

Doing surgery in $S_{b}$ with $D$ gives two spheres $S^{\prime}$ and $S^{\prime \prime}$, both spheres meet $k_{b}$ in two points, but at least one of them separates $k_{b}$ in two nontrivial parts, since otherwise $S_{b}$ would separate $k_{b}$ in two parts, one of them a trivial arc; but this is not possible because $S^{\prime}$ and $S^{\prime \prime}$ have less intersection circles with $\partial B$ than $S_{b}$ (see Figure 5). This completes the proof.

2.3. We construct a graph in $S_{a}$ as follows: the vertex set is formed by the $a_{i}$ 's, and the edges are the intersection arcs between $P_{a}$ and $P_{b}$. We denote the graph by $G_{a}$ (similarly $G_{b}$ ). The ends of each edge are labelled by some number; if the two labels are different, orient the edge from the higher label to the lower. We do not consider $a_{+}, a_{-}$as vertices, because there is no intersection arc incident to them. 
We define circuit, cycle, semicycle, source, sink, loop, unicycle, level edge, interior vertex, chord, label sequence, interior label, as in $\left[\mathbf{S}_{1}, 2.4\right]$ In addition, we allow an edge of a circuit to be a loop.

The interior of a circuit in $G_{a}$ is the component of its complement that does not contain $a_{-}$. A circuit of $G_{a}$ is bad if it contains $a_{+}$in its interior, otherwise it is good. A double loop is a circuit formed by two loops $c_{1}, c_{2}$ based at same vertex, and such that $c_{1}$ is in the interior of $c_{2}$.

2.4 LEMMA. (1) No chord of an innermost cycle or semicycle is oriented.

(2) If an innermost cycle or semicycle has an interior vertex it must have an interior source or sink.

(3) Any loop which has interior vertices has in its interior either a sink or source or a cycle.

(4) A semicycle with exactly one level edge has in its interior either a source or sink, or a cycle, or a loop, or a semicycle with exactly one level edge and without interior vertices or chords.

ProOF. It is similar to $\left[\mathbf{S}_{1}, 2.5\right]$.

2.5 LEMMA. $n_{a}>0$.

PROOF. If $n_{a}=0, S_{a}$ does not meet $\partial B$, hence it is contained in the interior of $B$; but $(B, t)$ is a prime tangle, and so $S_{a}$ is the boundary of a 3 -ball meeting $k_{a}$ in an unknotted spanning arc, contradicting the choice of $S_{a}$. This completes the proof.

\subsection{LEMMA. A good loop in $G_{a}$ has interior vertices.}

PrOOF. Suppose that in $G_{a}$ there is a good loop without interior vertices. Take an innermost such loop, let this be $\gamma$ based at $a_{i}$; its labels at $a_{i}$ are adjacent and are $j, j+1$, or $n_{b}, n_{b}$, or 1,1 (see Figure 4 ). In the first case the disc determined by the interior of $\gamma$ together with the annulus in $\partial B$ bounded by $b_{j}$ and $b_{j+1}$ can be used to obtain a compression disc for $P_{b}-\left\{b_{+}, b_{-}\right\}$, but this is not possible.

So suppose that the ends of $\gamma$ are labeled 1 (the remaining case is identical). Let $D$ be the disc in $S_{a}$ determined by the interior of $\gamma$, and let $\alpha$ be the arc of $a_{i}$ contained in the interior of $\gamma$, then $\partial D=\gamma \cup \alpha$. Consider the arc $\gamma$ in $G_{b}$, a loop based at $b_{1}$ with ends labeled $i$. Let $E$ be the disc in $S_{b}$ determined by the interior of $\gamma$ in $G_{b}$, and $\beta$ be the arc of $b_{1}$ contained in the interior of $\gamma$. Then $\partial E=\gamma \cup \beta$, $\alpha \cap \beta=\partial \alpha=\partial \beta=\partial \gamma$. There is disc $F$ properly embedded in $B^{\prime}$ with interior disjoint of $S_{b}$, and such that $\partial F=\alpha \cup \beta$, as in Figure $6 . k_{b}$ meets $D \cup F$ only in one point, this intersection occurs over $\alpha$. There are two subcases.

(a) $\gamma$ in $G_{b}$ is a good loop.

In this case $k_{b}$ does not meet $E$, so $D \cup E \cup F$ is a sphere which intersects $k_{b}$ in one point, but this is not possible.

(b) $\gamma$ in $G_{b}$ is a bad loop.

Let $E^{\prime}$ be the other disc in $S_{b}$ determined by $\gamma \cup \beta . \quad k_{b}$ intersects each one of $E$ and $E^{\prime}$ in one point. Doing surgery on $S_{b}$ with $D \cup F$ gives two spheres, $S=D \cup F \cup E$ and $S^{\prime}=D \cup F \cup E^{\prime}$ (Figure 7); each one of them meets $k_{b}$ in two points, and at least one of them, say $S$, must separate $k_{b}$ into two nontrivial parts (that is, none of the parts is an unknotted spanning arc), since otherwise $S_{b}$ 


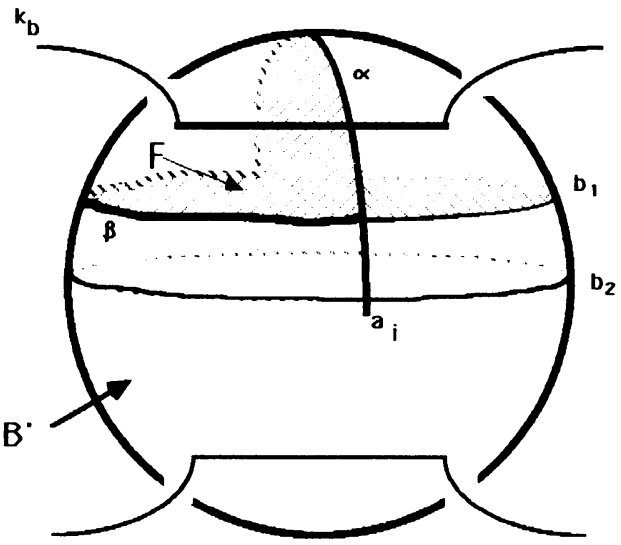

FIGURE 6

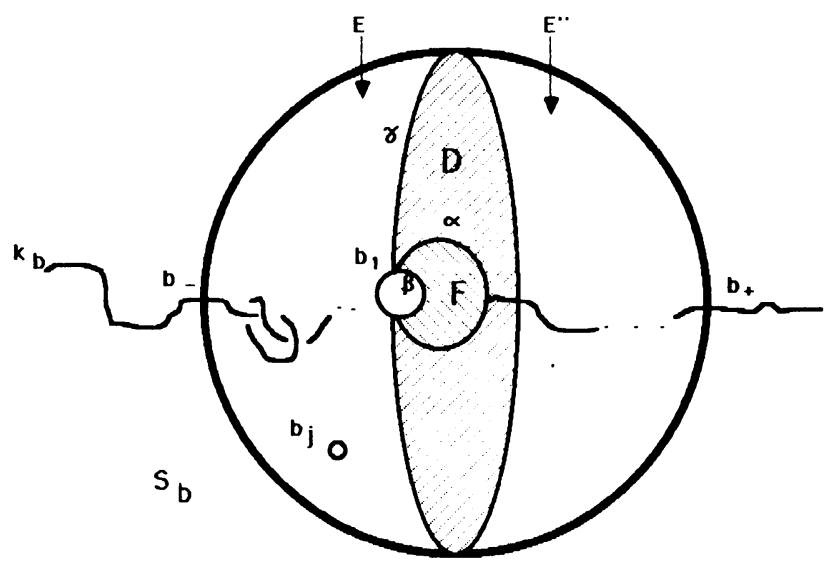

FIGURE 7

would separate $k_{b}$ so that one of the parts would be a trivial arc. After an isotopy we can suppose that $S \cap \partial B$ consists of essential circles in $\partial B$-\{strings of $\left.\left(B^{\prime}, r_{b}\right)\right\}$, and that $S \cap k_{b}$ lies in the strings of $(B, t)$. There are two such possible isotopies, choose the one which eliminates $b_{1}$, so that $\# \partial P \leq n_{b}-1(P=S \cap B)$, but this contradicts the minimality of \# $\partial P_{b}$. This completes the proof.

2.7 LEMMA. Let $c$ be a bad level loop in $G_{a}$ without vertices or edges in its interior. Then the corresponding level loop $c$ in $G_{b}$ is a good loop.

Proof. Suppose that the claim is false, that is, $c$ is also a bad loop in $G_{b}$. The ends of $c$ in $G_{a}$ are labeled $n_{b}$ or 1, w.l.o.g. suppose that they are labeled 1. $c$ in $G_{b}$ is a loop based at $b_{1}$. Let $D(E)$ be the disc in $S_{a}\left(S_{b}\right)$ determined by the interior of $c$ in $G_{a}\left(G_{b}\right) ; D \cap E=c$, because by 2.2 there is no intersection circle of $S_{a}$ and $S_{b}$ in $D$. As in the previous lemma, there is a disc $F$ properly embedded in $B^{\prime}$, with interior disjoint of $S_{b}$, such that $F \cap(D \cup E)=\partial F=\partial(D \cup E) . k_{b}$ meets the sphere $F \cup D \cup E$ in three points, one in the interior of $D\left(a_{+}\right)$, one in the interior of $E\left(b_{+}\right)$, and the other in $\partial F \cap \partial D$, but this is not possible, because $S^{3}$ is irreducible. This completes the proof. 
2.8 LEMMA. A semicycle in $G_{a}$ with neither chords nor interior vertices cannot have exactly one level edge.

PRoOF. See $\left[\mathbf{S}_{2}, 5.4\right]$.

2.9 LEMMA. An innermost cycle in $G_{a}$ with more than one edge has interior vertices.

PROOF. Suppose this is false, let $c$ be an innermost cycle without interior vertices. Let $D$ be the disc determined by the interior of $c$; by 2.4 there is no oriented edge in $D$, and an application of 2.8 shows that there is no level edge in $D$; so there is no edge in $D$, and by 2.2 there is no intersection circle between $S_{a}$ and $S_{b}$ in $D$. Because $c$ has at least two edges we can construct a punctured lens space as in $\left[\mathbf{S}_{2}, 5.6\right]$, even if $a_{+}$is in $D$. But this is not possible. Therefore the only cycles that may have no interior vertices are those cycles which have a bad unicycle in its interior.

2.10 LEMMA. Let $c$ be a cycle or a loop in $G_{a}$, then either

(a) c has in its interior a source or sink at which no loop is based; or

(b) $c$ is or $c$ has in its interior a bad level loop without chords or interior vertices; or

(c) $c$ is or $c$ has in its interior a bad unicycle without chords or interior vertices.

PROOF. Take a cycle or loop $\sigma$ contained in the interior of $c$, such that $\sigma$ has no cycle or loop in its interior. If $\sigma$ is a good loop or a cycle with more than two edges, then by 2.6 and $2.9 \sigma$ has vertices in its interior, and by 2.4 it has a source or sink in its interior, the election of $\sigma$ implies this source or sink has no loops. So we have (a) unless $\sigma$ is a bad loop. If $\sigma$ is a bad loop but it has interior vertices, then again by 2.4 and the election of $\sigma$, there is a source or sink where no loop is based. If $\sigma$ has no interior vertices, then $\sigma$ has no chords, and $\sigma$ is oriented or level, so we have (b) or (c). This completes the proof.

2.11 LEMMA. If $G_{a}$ has a bad level loop without chords or interior vertices, then $G_{b}$ has a source or sink at which no loop is based.

Proof. Let $c$ be this loop in $G_{a}$, by 2.7, $c$ in $G_{b}$ is a good loop. By $2.10 c$ in $G_{b}$ must have an interior source or sink at which no loop is based, because as $c$ is a good loop, (b) and (c) of 2.10 cannot happen. This completes the proof.

Two edges in $G_{a}$ are parallel if they bound a disk in $P_{a}$, thus either they are loops based at the same vertex, or they join two distinct vertices, but in any case the circuit they form has no interior vertices.

2.12 LEMMA. Let $e_{1}, e_{2}, \ldots, e_{p}$ be parallel edges in $G_{a}$, then either

(a) each $e_{i}$ is level; or

(b) each $e_{i}$ is oriented.

If (b) then either

$\left(\mathrm{b}^{\prime}\right) p \leq n_{b}$; or

$\left(\mathrm{b}^{\prime \prime}\right)$ there are two edges $e_{i}, e_{j}$ that form a cycle.

PROOF. The edges $e_{1}, \ldots, e_{p}$ join $u$ and $v$ (possibly $u=v$ ); if some edge is oriented and the other is level, then there are two consecutive edges $e_{i}$ and $e_{i+1}$, $e_{i}$ is oriented and $e_{i+1}$ level, i.e. a semicycle with exactly one level edge and with 


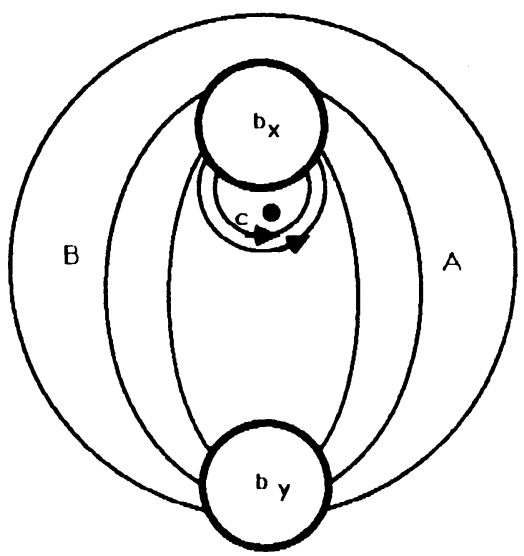

FIGURE 8

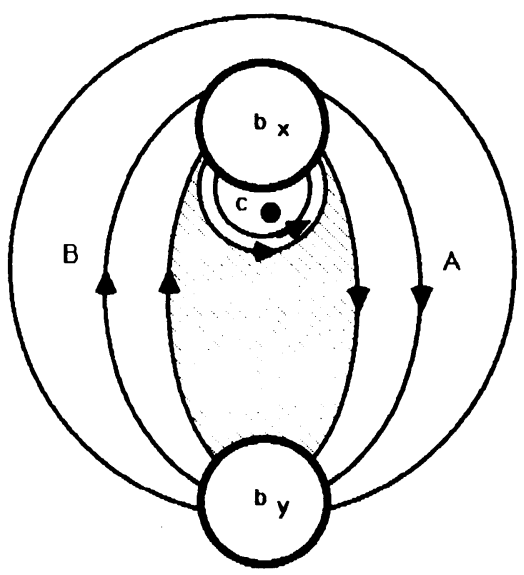

FIGURE 9

neither chords nor interior vertices, contradicting 2.8. Hence all the edges are level or all are oriented. Suppose that all the edges are oriented, that no two of them form a cycle, that $p>n_{b}$ and that the edges are oriented from $u$ toward $v$. Take a subset of them that consists of exactly $n_{b}+1$ consecutive edges, call them $e_{1}, \ldots, e_{n_{b}+1}$. Now the label of each $e_{i}$ at $u(v)$ must be greater than 1 (less than $n_{b}$ ), since if the contrary occurs some $e_{i}$ points toward $u$ (points away from $v$ ). If the labels of $e_{1}$ are $k$ and $s$ at $u$ and $v$ respectively, the labels of $e_{n_{b}+1}$ must be $n_{b}-k+1$ and $n_{b}-s+1$ at $u$ and $v$, therefore $k>s$ and $n_{b}-k+1>n_{b}-s+1$, that is $k>s$ and $k<s$, but this is not possible. This completes the proof.

2.13 LEMMA. For each vertex $v$ in $G_{a}$ there is an $i, 1 \leq i \leq n_{b}$, such that all the edges at $v$ with label $i$ are oriented. In particular $n_{b}>1$.

ProOF. Suppose this is false, then there is a vertex $v$ in $G_{a}$ such that for each $i, 1 \leq i \leq n_{b}$, there is a level edge adjacent to $v$ with label $i$. This implies that each vertex $b_{j}$ in $G_{b}$ is the base of a loop; if one of these loops is good, it is possible to find a good loop without interior vertices, contradicting 2.6; therefore suppose all the loops in $G_{b}$ are bad. Then there is a vertex $b_{x}$ in $G_{b}$ such that the loops there have no interior vertices.

If $n_{b}=1, b_{x}$ is the only vertex in $G_{b}$, and all the edges are bad loops, by 2.12 all these loops are level or all are oriented. If all the loops are level, in $G_{a}$ each vertex is the base of a loop and since in $G_{b}$ there is a bad level loop without chords or interior vertices, in $G_{a}$ there is a good loop by 2.7, and therefore it is possible to find a good loop in $G_{a}$ without interior vertices, contradicting 2.6; if all these loops are oriented, by 2.12 we must have a cycle in $G_{b}$, but this cycle has no interior vertices, contradicting 2.9 , therefore $n_{b}>1$.

As $n_{b}>1$ there is another vertex $b_{y}$ in $G_{b}$ such that the loops based there have only $b_{x}$ as an interior vertex, hence all the edges at $b_{x}$ are either loops or arcs joining $b_{x}$ and $b_{y}$. Let $c$ be an innermost loop based at $b_{x}$; there are two cases:

(1) $c$ is level.

By 2.7 the corresponding loop $c$ in $G_{a}$ is good, so it is sufficient to prove that for each $i, 1 \leq i \leq n_{a}$, there is incident to $b_{x}$ one level edge with label $i$, because this 


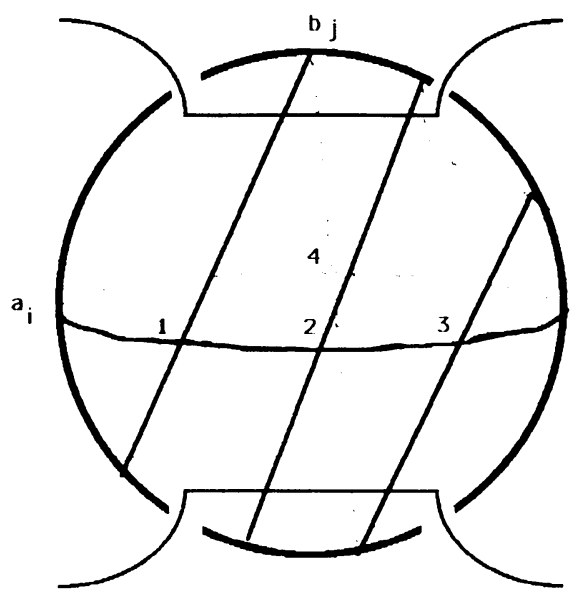

FIGURE 10

implies each vertex in $G_{a}$ is the base of a loop, and as there is a good loop, there will be a good loop without interior vertices, contradicting 2.6.

The labels of $c$ in $b_{x}$ are 1,1 or $n_{a}, n_{a}$. If there are $n_{a}$ or more loops in $b_{x}$, for each $i$ there will be a level loop based at $b_{x}$ with labels $i$. If there are less than $n_{a}$ loops in $b_{x}$, there are at least $2 n_{a}+2$ edges connecting $b_{x}$ and $b_{y}$; there are one or two sets of parallel edges, $A$ and $B$, connecting $b_{x}$ and $b_{y}$ (see Figure 8); one of these sets, say $A$, has at least $n_{a}+1$ edges, by 2.12 these edges are level, otherwise there is a good cycle without interior vertices; the labels of the edges of $A$ and the labels of the loops in $b_{x}$ are consecutive, so we have for each label $i, 1 \leq i \leq n_{a}$, at least one level edge with label $i$ in $b_{x}$.

(2) $c$ is oriented.

By 2.12 there are at most $n_{a}$ oriented loops in $b_{x}$. Let $r=d\left(r_{1}, r_{2}\right)$. There are at least $2(r-1) n_{a}$ edges connecting $b_{x}$ and $b_{y}$, hence one or two sets of parallel edges, $A$ and $B$, connecting $b_{x}$ and $b_{y}$ (see Figure 8 ). If one of these sets has more than $n_{a}$ edges, these edges must be level by 2.12. Suppose $r \geq 3$, so $|A \cup B| \geq 4 n_{a}$; if $|A|>n_{a}$ and $|B|>n_{a}$, these edges are level; if $|A| \geq 3 n_{a}$ and $|B| \leq n_{a}$, the edges of $A$ are level and the edges of $B$ may or may not be level; anyway there are at least $3 n_{a}$ level edges connecting $b_{x}$ and $b_{y}$, and for each $i, 1 \leq i \leq n_{a}$, there is at least one level edge with label $i$ connecting these vertices. Then in $G_{a}$ each vertex is the base of an oriented loop with labels $x$ and $y$, so all the loops in $G_{a}$ are bad. All the loops based on a vertex in $G_{a}$ are parallel, so there are at most $n_{b}$ loops at each vertex of $G_{a}$, so there are at most two loops based in a vertex with labels $x, y$. As there are at least $3 n_{a}$ level edges connecting $b_{x}$ and $b_{y}$ which have consecutive lables in $b_{x}$, there is at least one label $i$, such that there are three level edges with label $i$ connecting $b_{x}$ and $b_{y}$. Then $a_{i}$ in $G_{a}$ is the base of three loops with labels $x, y$, but this is a contradiction.

Suppose now $r=2$. We wish to prove that for each $i, 1 \leq i \leq n_{a}$, there is one level edge with label $i$ connecting $b_{x}$ and $b_{y}$, and that for the labels $1, n_{a}$ there are two such level edges. 
There are at most $n_{a}$ loops at $b_{x}$. Suppose with no loss of generality the winding number of these loops with respect to $a_{+}$is 1 . There are at least $2 n_{a}$ edges connecting $b_{x}$ and $b_{y}$, all these edges can be oriented only if $|A|=|B|=n_{a}$, by 2.12 , and in this case there are $n_{a}$ loops at $b_{x}$. Suppose these edges are oriented. We have a situation like in Figure 9; at the right of $c$ must be an edge $e_{a}$ of $A$ with label $n_{a}$ in $b_{x}$, and at left of $c$ must be an edge $e_{b}$ of $B$ with label 1 in $b_{x}$. Then $e_{a}\left(e_{b}\right)$ is oriented from $b_{x}$ into $b_{y}\left(b_{y}\right.$ into $\left.b_{x}\right)$; a good cycle is formed with these edges and one loop in $b_{x}$, like in Figure 9, but this cycle does not have interior vertices, which is a contradiction.

So suppose the edges of $A$ are level (the other case is similar). If the edges of $B$ are level we are finished. So suppose the edges of $B$ are oriented; these edges must be oriented from $b_{x}$ into $b_{y}$, otherwise there would be a semicycle with exactly one level edge and without interior vertices or chords. There are four labels 1 in $b_{x}$, these labels cannot be ends of edges at $B$ because these edges are oriented from $b_{x}$ to $b_{y}$, and at most two of these labels are ends of the loops at $b_{x}$, so at least two of these labels are ends of edges of $A$. There are also two labels $n_{a}$ in the end of edges of $A$, because of existence of the labels 1 in $A$ and the orientation of the loops. So for each label $i$, there is one level edge with labels $i$ connecting $b_{x}$ and $b_{y}$, and there are two such level edges with label $1, n_{a}$ (these edges may not be parallel). Then each vertex in $G_{a}$ is the base of a loop, and all these loops are bad.

Label the four points of intersection between $a_{i}$ and $b_{j}$ as $j_{1}, j_{2}, j_{3}, j_{4}\left(i_{1}, i_{2}, i_{3}, i_{4}\right)$ in $a_{i}\left(b_{j}\right)$, so that $a_{i}$ runs through them in the cyclic order $j_{1}, j_{2}, j_{3}, j_{4}$. The full set of labels in $a_{i}$ is $1_{1}, 2_{1}, \ldots, n_{b_{1}}, n_{b_{2}}, \ldots, 1_{2}, 1_{3}, \ldots, 1_{4}$. Observe that $b_{j}$ runs through the labels $i_{k}$ in the cyclic order $i_{1}, i_{2}, i_{3}, i_{4}$, or its inverse, as is shown in Figure 10. If an edge $\alpha$ in $G_{a}$ connects $a_{i}$ and $a_{k}$ with labels $j_{s}$ and $g_{t}$ respectively, then the corresponding edge $\alpha$ in $G_{b}$ connects $b_{j}$ and $b_{g}$ with labels $i_{s}$ and $k_{t}$ respectively.

Consider only the vertices $b_{x}, b_{y}, a_{1}, a_{n_{a}}$. The labels of $a_{i}$ when it meets $b_{x}$ and $b_{y}$ are ordered as follows: $x_{1}, x_{2}, y_{2}, y_{3}, x_{3}, x_{4}, y_{4}, y_{1}$, or $x_{1}, y_{1}, y_{2}, x_{2}, x_{3}, y_{3}, y_{4}, x_{4}$. The labels in $b_{x}$ and $b_{y}$ are ordered as $1_{1}, 1_{2}, n_{a_{2}}, n_{a_{3}}, 1_{3}, 1_{4}, n_{a_{4}}, n_{a_{1}}$, or $1_{1}, n_{a_{1}}$, $n_{a_{2}}, 1_{2}, 1_{3}, n_{a_{3}}, n_{a_{4}}, 1_{4}$, but equal or inverse in both $b_{x}$ and $b_{y}$. There are two bad loops in $a_{1}$ with labels $x, y$, these loops have the same orientation (otherwise they form a good cycle without interior vertices), so we use exactly three subindices (e.g. 1, 2, 2, 3). The corresponding edges to these loops in $G_{b}$ are two level edges connecting $b_{x}$ and $b_{y}$ with labels 1 , furthermore we can suppose the labels 1 are adjacent in $b_{x}$. If also in $b_{y}$ the ends are adjacent, we are using two or four subindices (e.g. 1, 2 in both $b_{x}$ and $b_{y}$, or 1,2 in one and 3,4 in the other), but this is a contradiction. Now if the ends of these edges are not adjacent in $b_{y}$, then the ends of the two level edges with label $n_{a}$ are adjacent in both $b_{x}$ and $b_{y}$, so using $n_{a}$ instead of 1 and repeating the argument, a contradiction is obtained; this is shown in Figure 11. This completes the proof.

2.14 LEMMA. Let $v$ be a vertex of $G_{a}$, suppose there is a family $A$ of consecutive oriented edges that point into $v$, and a family $B$ of consecutive oriented edges that point out from $v$; furthermore the last edge of $A$ and the first of $B$ (or vice versa) are adjacent at $v$. Then there is a set $\mathcal{L}=\left\{1, \ldots, n_{b}\right\}$ of $n_{b}$ consecutive labels of $v$ at which no edge of $A \cup B$ is incident, and the label $1\left(n_{b}\right)$ is closer than $n_{b}(1)$ to the labels of $B(A)$ (that is, there is an arc of $v$, with interior disjoint from $\mathcal{L}$, $A$ and $B$ joining $1\left(n_{b}\right)$ to a label of $\left.B(A)\right)$. 

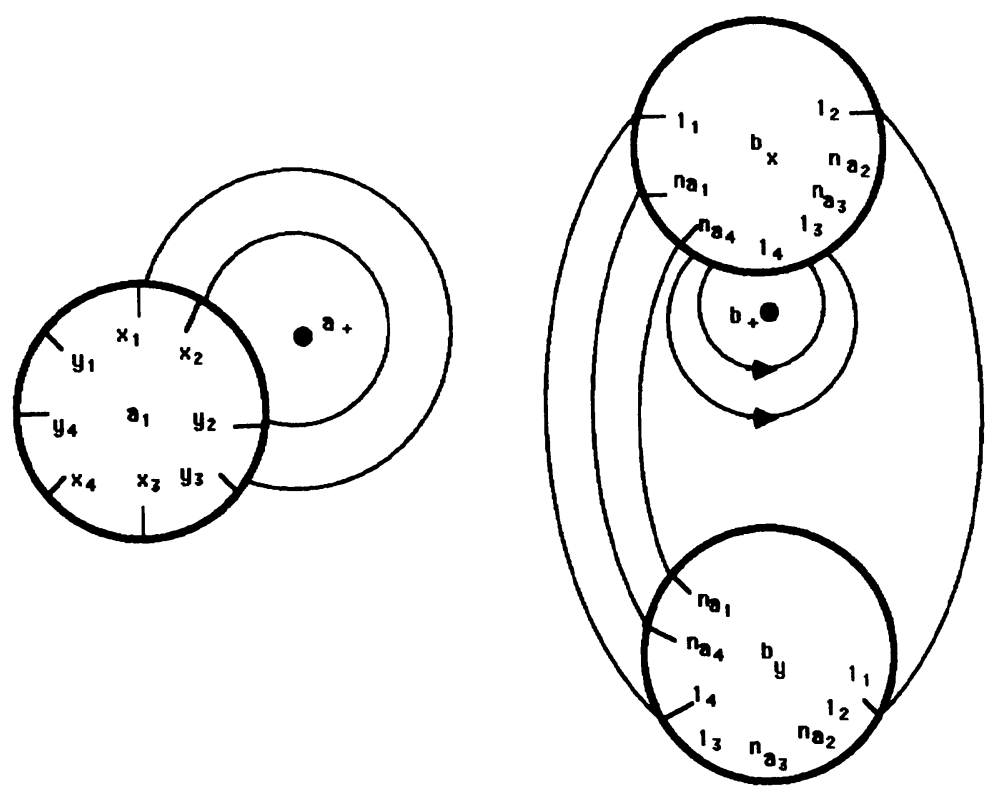

FIGURE 11

PROOF. Suppose with no loss of generality we have a situation as in Figure 12. Go through the labels of $v$ in the counterclockwise direction and pick up the first label $n_{b}$ (denote it by $n_{b}^{*}$ ) found after crossing the labels of $A$. Consider the set $\mathcal{K}=\left\{n_{b}^{*}, \ldots, 1,1, \ldots, n_{b}, n_{b}, \ldots, 1\right)$ of $3 n_{b}$ consecutive labels of $v$, beginning with $n_{b}^{*}$, going in the clockwise direction. The ends of $A(B)$ in $v$ cannot be labeled with $n_{b}(1)$, due to its orientation. So the labels of $A$ in $v$ are contained in the portion $n_{b-1}, \ldots, 1,1, \ldots, n_{b-1}$ of $\mathcal{K}$, and the labels of $B$ in the portion $n_{b-2}, \ldots, 2$ or in $2, \ldots, n_{b}, n_{b}, \ldots, 2$; so the labels of $A \cup B$ are contained in $\mathcal{K}$. Take the set $\mathcal{L}=\left\{1, \ldots, n_{b}\right\}$ of $n_{b}$ consecutive labels of $v$, which is after $\mathcal{K}$ going in the clockwise direction. $\mathcal{L}$ does not overlap $\mathcal{K}$ because $r \geq 2$. Clearly $\mathcal{L}$ has the desired properties. This completes the proof.

2.15 LEMMA. Let $v$ be a vertex in $G_{a}$ at which is based a bad unicycle without interior vertices. Then in $G_{a}$ there is a source or sink where no loops are based.

PROOF. All the loops based at $v$ without interior vertices are oriented and parallel. Let $c_{1}$ be the bad unicycle without interior vertices or chords based at $v$. There are at most $n_{b}$ bad unicycles based at $v$ without interior vertices, let these be $c_{1}, c_{2}, \ldots, c_{m}$. We can suppose there is no good loop in $G_{a}$, because if there is one by 2.10 we finish. Suppose that there is another bad loop, say $c^{\prime}$, based at $u$, so that there are no loops other than $c_{1}, \ldots, c_{m}$ in its interior. Let $D$ be the interior of $c^{\prime}$. If $v \neq u$ an analogous argument to that of 2.14 shows that there are vertices in $D$ other than $v$; if $v=u$ the choice of $c^{\prime}$ implies there are vertices in $D$. If there is no loop other than $c_{1}, \ldots, c_{m}$ the proof is similar.

If there is a good cycle in $D$ we are finished (by 2.10), so suppose all the cycles in $D$ are bad. Let $C$ be the set of all the bad cycles in $D$ that have no interior vertices. Note that $c_{1}$ is in $C, v$ is a vertex of each one of these cycles, and all 


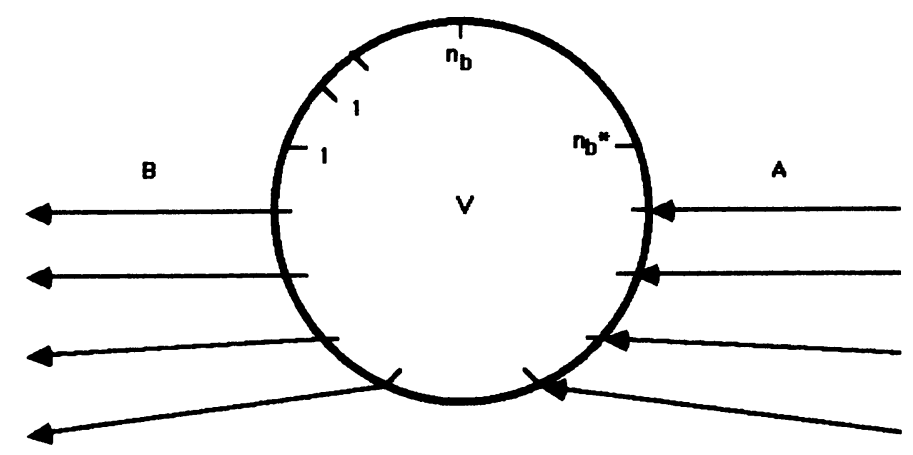

FIGURE 12

these cycles have the same winding number with respect to $a_{+}$as $c_{1}$. We have a situation as in Figure 13. Let $H$ be the subgraph of $G_{a}$ defined as follows: \{vertices of $H\}=\left\{\right.$ vertices of $G_{a}$ which are in $D(u$ included $\left.)\right\}$, edges of $\left.H\right\}=\left\{\right.$ edges of $G_{a}$ which are in $D$ except the edges of the cycles of $C$ \}. We have two cases.

(1) There is no source or sink in $H$ (except possibly $u$ ).

Because there is at most one source or sink in $H$ (but not both), there are cycles in $H$. Take one innermost, let this be $\sigma$. By the selection of $C, \sigma$ has interior vertices, and by $2.4 \sigma$ has an interior source or sink, $u$ cannot be in the interior of $\sigma$, so this is a contradiction.

(2) There is a source or sink in $H$ (other than $u$ ).

If one vertex of $H$ which is not a vertex of the cycles of $C$ is a source or sink in $H$ we are finished, so suppose none of these vertices is a source or sink. Suppose with no loss of generality that there is a vertex $x$ in $H$, such that $x$ is a source in $H$, and it is a vertex of the cycles of $C$. Let $A(B)$ be the set of edges that belongs to the cycles of $C$ which point into (out of) $x$. It is not difficult to see that the sets $A, B$ satisfy the hypothesis of 2.14 ; so there is a set $\mathcal{L}=\left\{1, \ldots, n_{b}\right\}$ of consecutive labels of $x$ at which edges of $H$ are incident, and the label $1\left(n_{b}\right)$ is closer than $n_{b}$ (1) to the labels of $B(A)$. Because $x$ is a source in $H$, a level edge is incident to the label 1 , let this be $e_{x}^{\prime}$. By 2.13 there is a label $i$ in $\mathcal{L}$ at which is incident an oriented edge, let this be $e_{x}$, this edge point out of $x$. We can suppose we have a situation as in Figure 14, so that the winding number of $c_{1}$ with respect to $a_{+}$is 1 , and $e_{x}^{\prime}$ is at the left of $e_{x}$.

Construct a path $\gamma$ in $H$, starting with $e_{x}$, through oriented edges always consistent with its orientations. Finish the path when a vertex is repeated or when $\gamma$ reaches $u$ or a vertex of the cycles of $C$. Construct another path $\gamma^{\prime}$ in $H$, starting with $e_{x}^{\prime}$, through oriented edges (except $e_{x}^{\prime}$ ) always inconsistent with its orientations. Finish the path when a vertex is repeated, or when $\gamma^{\prime}$ reaches $u$, or a vertex of $\gamma$, or a vertex of the cycles of $C$. We have the following cases.

(a) The path $\gamma$ repeats a vertex.

Then a cycle $\sigma$ is formed, this cycle must be a bad one and contain all the vertices of the cycles of $C^{\prime}$ in its interior. There is a path $\sigma^{\prime}$ which joins $e_{x}$ with $\sigma$ $\left(\sigma \cup \sigma^{\prime}=\gamma\right)$. Consider the path $\gamma^{\prime}$, if $\gamma^{\prime}$ finishes at a vertex of $\gamma$ or at a vertex of the cycles of $C$, then with the path $\gamma^{\prime}$, a part of an outermost cycle of $C$ (possibly 
empty), and a part of $\gamma$ (possibly empty) a good semicycle in $G_{a}$ with exactly one level edge is formed; this is ensured by the existence of $\sigma^{\prime}$ (see Figure 14). No vertex of the cycles of $C$ is in the interior of this semicycle. So by 2.4 there is a good semicycle with exactly one level edge and without interior vertices or chords, but this contradicts 2.8 . If the path $\gamma^{\prime}$ repeats a vertex, then a good cycle or a good semicycle with exactly one level edge is formed (this is ensured by the existence of $\left.\sigma^{\prime}\right)$. the same argument as above yields a contradiction.

(b) $\gamma$ finishes at $u$.

The same argument as in case (a) yields a contradiction.

(c) $\gamma$ finishes at a vertex of the cycles of $C$.

$\gamma$ together with a part of a cycle of $C$ form a cycle in $G_{a}$, this cycle either is good or it is bad and contains $e_{x}^{\prime}$ in its interior, now we proceed as in case (a).

In the above argument it was important that $e_{x}^{\prime}$ be at the left of $e_{x}$, because if $e_{x}^{\prime}$ had been at the right of $e_{x}$, then no contradiction would be obtained. This completes the proof.

\subsection{LEMMA. $G_{a}$ or $G_{b}$ has a source or sink where no loop is based.}

PROOF. By 2.13 there are oriented edges in $G_{a}$; if $G_{a}$ has no cycles or loops, then there is a source or sink with the desired properties. If there is a cycle or a loop in $G_{a}$, then by $2.10,2.11$, and 2.15 there is a source or $\operatorname{sink}$ in $G_{a}$ or $G_{b}$ where no loop is based. This completes the proof.

Let $p$ be an integer, $1 \leq p \leq n_{b}$, define a $p$-biflow to be a circuit in $G_{a}$ with the following properties:

(a) All edges are oriented, with heads (tails) labeled $p$.

(b) All interior labels are integers greater than (less) $p$.

(c) There is precisely one vertex of the circuit (called the base) for which both incident edges point out (in) and one (called the apex) for which both incident edges point in (out).

(d) There are interior labels at the apex, in fact at least two.

This definition is equal to that of $\left[\mathbf{S}_{2}, 4.4\right]$, except by the property (d), this property is necessary for the proof of 2.17 . Define a $p$-loop to be a loop with one end labeled $p$ and either all interior labels greater than, or all less than $p$. Define a $p$-double loop to be a double loop that is a cycle and such that the two edges have heads (tails) labeled $p$ and all interior labels greater than (less than) $p$.

2.17 LEMMA. Suppose that $b_{p}$ is a source or sink in $G_{b}$ and $c$ is either a good $p$-biflow, or a good $p$-loop, or a good $p$-double loop in $G_{a}$, then in the interior of $c$ there is a $p$-loop or a $p$-biflow.

PROOF. It is similar to that of $\left[\mathbf{S}_{2}, 6.2,6.3\right]$.

$2.18 \mathrm{LEMMA}$. If $b_{p}$ is a source or sink in $G_{b}$, then in $G_{a}$ there are neither good p-biflows nor good p-loops nor good p-double loops.

PROOF. If there is one of these circuits, there is an innermost, but this contradicts 2.17. This completes the proof.

2.19 LEMmA. Suppose that $b_{p}$ is a source or sink in $G_{b}$ at which no loop is based, then in $G_{a}$ there is either a good $p$-loop or a good $p$-biflow or a good p-double loop. 


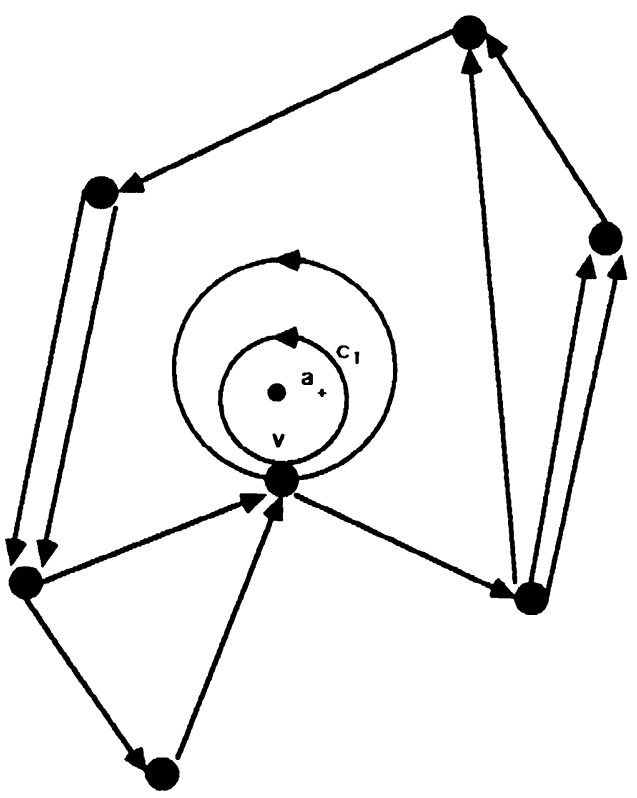

FIGURE 13

Proof. It is essentially equal to $\left[\mathbf{S}_{2}, 6.7\right]$.

The contradictions between the Lemmas 2.16, 2.18 and 2.19 complete the proof of Theorem 1.

3. Further applications of combinatorial techniques. I. Now we prove Theorem 2. Let $(B, t)$ be a prime tangle, $\left(B^{\prime}, r_{1}\right)$ and $\left(B^{\prime}, r_{2}\right)$ two rational tangles such that $r=d\left(r_{1}, r_{2}\right) \geq 2$. Let $k_{1}$ be the knot or link obtained by summing $(B, t)$ and $\left(B^{\prime}, r_{1}\right)$, suppose that $k_{1}$ is composite. There is a $S^{2}$ that meets $k_{1}$ in two points, such that neither of the closures of the two components of $S^{3}-S^{2}$ meets $k_{1}$ in an unknotted spanning arc. Suppose that $k_{2}$ is not a splittable link, we consider this case in $\S 4$. As in the previous section suppose that: (a) the strings of $\left(B^{\prime}, r_{1}\right)$ are contained in $\partial B^{\prime}$; (b) $S^{2}$ meets $k_{1}$ on the strings of $(B, t)$; (c) the intersections of $S^{2}$ and $\partial B$ are all essential circles in $\partial B$-\{strings of $\left.\left(B^{\prime}, r_{1}\right)\right\}$, such that each of these circles is the boundary of a disk in $S^{2}$ whose interior does not meet $\partial B$. Let $S_{1}$ be a sphere in $S^{3}$, with the above-mentioned properties such that the number of intersection circles between it and $\partial B$ is minimized.

Let $k_{2}$ be the link obtained by summing $(B, t)$ and $\left(B^{\prime}, r_{2}\right)$, suppose that $k_{2}$ is a splittable link, that is there is a $S^{2}$ disjoint of $k_{2}$ that separates the components of $k_{2}$. As before suppose that the strings of $\left(B^{\prime}, r_{2}\right)$ are on $\partial B$ and that the intersection circles between $S^{2}$ and $\partial B$ are essential in $\partial B$-\{strings of $\left(B^{\prime}, r_{2}\right)$ \}, and each of these circles is the boundary of a disk in $S^{2}$ whose interior does not meet $\partial B$. Let $S_{2}$ be a sphere as above which minimizes the number of intersection circles with $\partial B$.

Let $P_{1}=S_{1} \cap B$ and $P_{2}=S_{2} \cap B$, these are planar surfaces in $B . \partial P_{1}$ is formed by $n$ circles denoted by $a_{1}, \ldots, a_{n}$, parallel to $h_{r_{1}}(J)$, labeled so that $a_{i}$ and $a_{i+1}$ cobound an essential annulus in $\partial B$-\{strings of $\left.\left(B^{\prime}, r_{1}\right)\right\}$ whose interior does not 


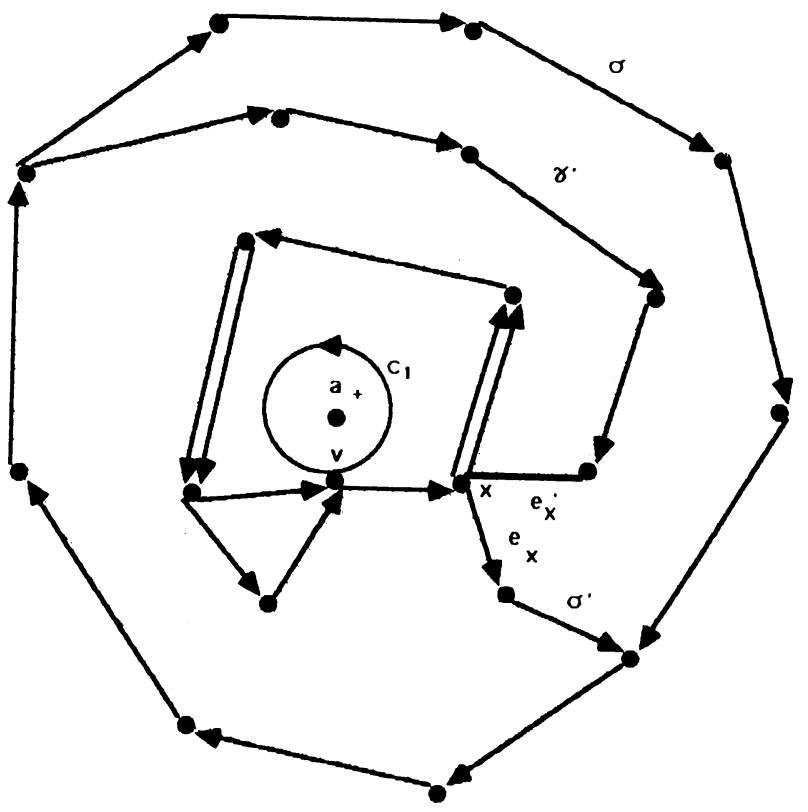

FIGURE 14

meet $S_{1}$, for $1 \leq i \leq n-1$. $\partial P_{2}$ is formed by $m$ circles denoted by $b_{1}, \ldots, b_{m}$, parallel to $h_{r_{2}}(J)$, and labeled as in $P_{1}$. Furthermore $m$ is odd. Denote the points of intersection between $P_{1}$ and $k_{1}$ by $a_{+}$and $a_{-}$. The way that an $a_{i}$ meets the $b_{j}$ 's is similar to that of $\S 2.1$ (see Figure 4).

$P_{1}$ and $P_{2}$ are incompressible in $B$-\{strings of $\left.(B, t)\right\}$, hence we can suppose that all the intersection circles between $P_{1}$ and $P_{2}$ are essential in both $P_{1}-\left\{a_{+}, a_{-}\right\}$ and $P_{2}$.

We construct graphs in $S_{1}$ and $S_{2}$ as before; the vertices are the $a_{i}$ 's and the $b_{j}$ 's respectively, and the edges are the intersection arcs between $P_{1}$ and $P_{2}$. Denote the graphs by $G_{1}$ and $G_{2}$. Label the ends of the edges and orient them as in the previous section.

The interior of a circuit in $G_{1}$ is the component of the complement of this circuit that does not contain $a_{-}$. A circuit in $G_{1}$ is good if it does not contain $a_{+}$in its interior. Take a point $x \in P_{2}-P_{1}$, define the interior of a circuit in $G_{2}$ to be the component of the complement of this circuit that does not contain $x$.

We have the following facts:

(1) $n>0$.

(2) A loop (good loop) in $G_{2}\left(G_{1}\right)$ has interior vertices.

(3) A cycle (good cycle) in $G_{2}\left(G_{1}\right)$ has interior vertices.

The proofs of these facts are similar to those of $\S 2$.

(4) There are oriented edges in $G_{2}$. If all the edges of $G_{2}$ are level, then in $G_{1}$ all the edges are loops. All of them are bad loops, otherwise there is a good loop without interior vertices. Take any vertex in $G_{1}$, all the edges incident to it are bad loops; if they are level then in $G_{2}$ each vertex is the base of a loop, so there is a loop without interior vertices, a contradiction. If all the loops are oriented, then 
because there are at least $2 m$ loops, by 2.12 two of them form a good cycle with no interior vertices, a contradiction.

An easy application of these facts show that in $G_{2}$ there is a source or sink at which no loop is based. The Lemmas $2.17,2.18$, and 2.19 can be applied without difficulty in this case. In those Lemmas $G_{1}$ plays the role of $G_{a}$ and $G_{2}$ the role of $G_{b}$.

This proves Theorem 2. The proof of this theorem is easier than the earlier one because in $G_{2}$ there are no bad circuits.

4. Further applications of combinatorial techniques. II. In this section we prove Theorem 3 . Let $(B, t)$ be any tangle, $\left(B^{\prime}, r_{1}\right)$ and $\left(B^{\prime}, r_{2}\right)$ two rational tangles. Suppose that summing $(B, t)$ to $\left(B^{\prime}, r_{i}\right), i=1,2$, gives a link $k_{i}$, which is splittable. Suppose $r_{1} \neq r_{2}$, so we have $d\left(r_{1}, r_{2}\right) \geq 2$ (any rational tangle to distance 1 of $\left(B^{\prime}, r_{1}\right)$ will give a knot when summing to $\left.(B, t)\right)$. We use the indices $a, b$ to denote 1 or 2 , as in $\S 2$.

As $k_{a}$ is splittable, there is a $S^{2}$ that does not meet $k_{a}$ and that separates the components of $k_{a}$. As in the previous sections we can suppose the following: The strings of $\left(B^{\prime}, r_{a}\right)$ are on $\partial B$; the intersections of $S^{2}$ and $\partial B$ are all essential circles in $\partial B$-\{strings of $\left.\left(B^{\prime}, r_{a}\right)\right\}$, such that each one of these circles is the boundary of a disk in $S^{2}$ whose interior does not meet $\partial B$. Let $S_{a}$ be a sphere as above which minimizes the number of intersections circles with $\partial B$.

Let $P_{a}=S_{a} \cap B$, this is a planar surface. $\partial P_{a}$ is formed by $n_{a}$ circles denoted by $a_{1}, \ldots, a_{n_{a}}$, parallel to $h_{r}(J)$, labeled so that $a_{i}$ and $a_{i+1}$ cobound an essential annulus in $\partial B$-\{strings of $\left.\left(B^{\prime}, r_{a}\right)\right\}$ whose irterior does not meet $S_{a}$, for $1 \leq i \leq$ $n_{a}-1$. Both $n_{a}$ and $n_{b}$ are odd. The way that an $a_{i}$ meets the $b_{j}$ 's is similar to that of $\S 2.1$, as in Figure 4.

$P_{a}$ is incompressible in $B$-\{strings of $\left.(B, t)\right\}$. We construct a graph $G_{a}$ in $P_{a}$, as before. Take a point $x \in P_{a}-P_{b}$, define the interior of a circuit in $G_{a}$ to be the component of the complement of this circuit that does not contain $x$.

We have the following facts: A loop in $G_{a}$ has interior vertices; a cycle in $G_{a}$ has interior vertices; there are oriented edges in $G_{a}$. The proofs of these facts are similar to the proofs of the previous sections. An easy application of those facts show that in $G_{a}$ there is a source or sink at which no loop is based. Let $v$ be a source ( $\operatorname{sink}$ ) in $G_{a}$ at which no loop is based, all the edges incident to $v$ with label $1\left(n_{b}\right)$ are level, therefore in $G_{b}, b_{1}\left(b_{n}\right)$ is the base of several loops, all with one label $i$. An innermost such loop will be a $i$-loop. Lemma 2.18 can be applied in the present case, and hence we find a contradiction. This completes the proof of Theorem 3.

I would like to express my sincerest gratitude to J. C. Gomez Larrañaga for his supervision of this work which formed part of my Masters thesis at U.N.A.M. I would like to thank M. Scharlemann for his discovery of an error in one of the lemmas. I am grateful to $\mathrm{H}$. Short and the referee for their suggestions.

\section{REFERENCES}

[B] S. A. Bleiler, Prime tangles and composite knots, Knot Theory and Manifolds, Lecture Notes in Math., vol. 1144, Springer-Verlag, Berlin and New York, 1985, pp. 1-13.

[BS 1 S. A. Bleiler and M. Scharlemann, Tangles, property $P$, and a problem of J. Martin, Math. Ann. 273 (1986), 215225. 
$\left[\mathbf{B S}_{2}\right]$, A projective plane in $\mathbf{R}^{4}$ with three critical points is standard, MSRI preprint.

[C] J. H. Conway, An enumeration of knots and links, and some of their algebraic propertis, Computational Problems in Abstract Algebra, Pergamon Press, Oxford and New York, 1969, pp. 329-358.

[E] M. Eudave-Muñoz, Cirugía en nudos fuertemente invertibles, An. Inst. Mat. Univ. Nac. Autónoma México 26 (1986), $41-57$.

[GL] C. McA. Gordon and J. Luecke, Only integral Dehn surgeries can yield reducible manifolds, preprint.

[KT] P. K. Kim and J. L. Tollefson, Splitting the P. L. involutions of nonprime 3-manifolds, Michigan Math. J. 27 (1980), 259274.

[L] W. B. R. Lickorish, Prime knots and tangles, Trans. Amer. Math. Soc. 267 (1981), 321-332.

$\left[\mathbf{M}_{1}\right]$ J. M. Montesinos, Variedades de Seifert que son recubridores cíclicos ramificados de dos hojas, Bol. Soc. Mat. Mexicana (2) 18 (1973), 1-32.

$\left[\mathbf{M}_{2}\right]$ _ Surgery on links and double branched covers of $S^{3}$, Ann. of Math. Studies, no. 84, Princeton Univ. Press, Princeton, N. J., 1975, pp. 227-260.

$\left[\mathbf{S}_{1}\right]$ M. Scharlemann, Smooth spheres in $\mathbf{R}^{4}$ with four critical points are standard, Invent. Math. 79 (1985), 125141 .

[S $\left.\mathbf{S}_{2}\right]$, Unknotting number one knots are prime, Invent. Math. 82 (1985), 37-55.

DepartieNt of MAThematics, UNiversity of California, SANTA Barbara, CALIFORNIA 93106 